\title{
Untersuchungen über die Differentialgleichung der hypergeometrischen Reihe $*$ ).
}

(Aus den hinterlassenen Papieren von C. G. J. Jacobi mitgetheilt durch Herrn E. Heine.)

\section{\$. 1.}

As ist seit Euler bekannt, dafs das bestimmte Integral

in welchem

$$
y=\int_{0}^{i 1} V d u \text {, }
$$

$$
V=u^{\beta-1}(1-u)^{\gamma-\beta-1}(1-x u)^{-\alpha}
$$

geselzt ist, der Differentialgleichung

$$
\text { (1.) } x(1-x) y^{\prime \prime}+(\gamma-(\alpha+\beta+1) x) y^{\prime}-\alpha \beta \gamma=0
$$

genügt. Um dies nach dem Vorgange Eulers (Institutiones calc. integr. Vol. II, Sect. I, Cap. X, Problema 130) zu beweisen, hat man nur nöthig, in die linke Seite von (1.) für $y$ das unbestimmte Integral $\int V d u$ zu setzen, durch Differentiation unter dem Integrale $y^{\prime}$ und $y^{\prime \prime}$, d. h. $\frac{d y}{d x}$ und $\frac{d^{2} y}{d x}$, zu bilden, und endlich zu reduciren. Dann erhält man auf der rechten Seite zu-

*) Die Abhandlung, die ich hier mittheile, verdankt ihre Entstehung einer Handschrift Jacobis, welche aus einer ziemlich frühen Zeit seines Lebens zu stammen scheint. Nachdem er aus dem reichen Inhalt derselben den Stoff eines Theils der Abhandlung: Formula transformationis integralium definitorum (Bd. 15 dieses Journals), sowie der $\mathbf{A b}$ handlung: Ueber die Entwicklung des Ausdrucks etc. (Bd. 26 dieses Journals oder Journal de M. Liouville année $1845 \mathrm{p} .229$ ) entnommen hatte, blieb ein noch unbenutzter Theil der Handschrift übrig, welcher die Kugelfunctionen betraf. Die in demselben enthaltenen Untersuchungen wurden von Jacobi im Jahre 1843, indem er sie einer neuen Bearbeitung unterwarf, auf die allgemeine hypergeometrische Reihe ausgedehnt, und ich übernahm es während meiner damaligen Studienzeit, die in solcher Art verallgemeinerten und zu späterer Veröffentlichung bestimmten Ergebnisse zu einem Aufsatz zusammenzustellen, dessen Herausgabe jetzt in seiner dem Wesen nach unveränderten Form erfolgt, nachdem eine von Jacobis Hand beabsichtigte Umgestaltung durch seinen Tod vereitelt worden ist. 
năchst nicht 0, sondern den Ausdruck *)

$$
-\alpha u^{\beta}(1-u)^{\gamma-\beta}(1-x u)^{-\alpha-1}=-\alpha \cdot \frac{u(1-u)}{1-x u} V .
$$

Da derselbe für $u=0$ und $u=1$ verschwindet, natürlich $\beta$ und $\gamma-\beta$ positiv gedacht, so wird das bestimmte Integral $y=\int_{0}^{1} V d x$, wenn es einen Sinn hat, (1.) integriren.

Es ist früher nicht beachtet worden, dafs der erwähnte Ausdruck auch für $u= \pm \infty$ verschwindet, wenn $\gamma-\alpha-1$ negativ ist, so dafs alsdann aufser den Grenzen 0 und 1, auch die Grenzen 0 und $-\infty, 1$ und $\infty$ Integrale von (1:) verschaffen. Mit Hinzuziehung hiervon hat man das Resultat: Es genügt $y=\int_{g}^{h} V d u$ der Gleichung (1.), wenn $g$ und $h$ zwei von den Werthen $0,1, \pm \infty$ bezeichnen, und $\left[\frac{u(1-u)}{(1-x u)} V\right]_{g}^{h}=0$ ist; vorausgesetzt dafs das Integral eine Bedeutung hat. Wir bedienen uns der bekannten Bezeichnung, nach der $[f(u)]_{g}^{h}$ die Differenz $f(h)-f(g)$ vorstellt.

Mit Rücksicht auf die Zusammensetzung des Ausdrucks $V$ lag es nahe, zu den soeben bétrachteten Werthen der Grenzen des Integrals $\int V d u$, von denen die beiden ersten die Grölsen $u$ und $1-u$ gleich Null machen, den Werth $\frac{1}{x}$ hinzuzufügen, für welchen $1-x u$ verschwindet. Indem zunächst in die linke Seite von (1.) $y=\int_{g}^{\frac{\varepsilon}{x}} V d u$ gesetzt wurde, wo $\varepsilon$ eine Constante bedeutet, ergab sich nach gehöriger Reduction:

$$
\begin{aligned}
& -(\gamma-\beta-1) \varepsilon^{\beta}(1-\varepsilon)^{1-\alpha} x^{1-\gamma}(x-\varepsilon)^{\gamma-\beta-1} \\
& +\alpha g^{\beta}(1-g)^{\gamma-\beta}(1-x g)^{-\alpha-1}
\end{aligned}
$$

und hieraus wurde für $\varepsilon=1$ geschlossen, dafs auch

*) Dies kann man auch mit Euler so ausdrücken, dafs der vollständige nach $u$ genommene Differentialquotient

unter der Form

$$
-\alpha \frac{d}{d u}\left(\frac{u(1-u)}{1-x u} V\right)
$$

$$
A \frac{d^{2} V}{d x^{2}}+B \frac{d V}{d x}+C V
$$

darstellbar ist, wo $A, B, C$ die von $u$ unabhängigen in Gleichung (1.) vorkommenden Grölsen $x(1-x), \gamma-(\alpha+\beta+1) x,-\alpha \beta$ sind. 
der Gleichung (1.) genügt, wenn

$$
y=\int_{g}^{\frac{1}{x}} V d u
$$

$$
\frac{u(1-u)}{(1-x u)} \boldsymbol{V}
$$

für $u=g$ verschwindet, und $1-\alpha$ positiv ist; dafs das Integral einen Werth haben mufs, ist vorausgesetzt.

Man hat demnach sechs, als bestimmte Integrale auftretende und, wie leicht zu zeigen ist, verschiedene Lösungen der Gleichung (1.) (d. h. solche, von denen nicht zwei einen constanten Quotienten haben). Indem wir uns hier, wie auch im Folgenden, $x$ immer positiv denken, auf welchen Fall der eines negativen $\boldsymbol{x}$ leicht zurückzuführen ist, stellen' wir diese Lösungen mit Hinzufügung der Bedingungen, unter denen sie der Gleichung (1.) genügen, zusammen :

$$
\begin{aligned}
& \text { 1) wenn } \beta \text { und } \gamma-\beta \text { positiv ist: } y=\int_{0}^{1} V d u \text {, } \\
& \text { 2) }-\beta \quad-\alpha+1-\gamma-\quad-y=\int_{0}^{-\infty} V d u \text {, } \\
& \text { 3) }-\gamma-\beta-\alpha+1-\gamma--y=\int_{1}^{\infty} V \mathrm{~d} u \text {, } \\
& \text { 4) }-\beta-1-\alpha-2-y=\int_{0}^{\frac{1}{x}} V d u \text {, } \\
& \text { 5) }-\alpha+1-\gamma-1-\alpha-\quad-y=\int_{\frac{1}{x}}^{\infty} V d u \text {, } \\
& \text { 6) }-\gamma-\beta-1-\alpha-\quad-y=\int_{1}^{\frac{1}{x}} V d u \text {. }
\end{aligned}
$$

Um die Bedeutung dieser Integrale besser zu übersehen, kann man sie durch hypergeometrische Reihen ausdrücken. Man weifs nämlich, dafs $\int_{0}^{1} u^{\lambda}(1-u)^{\mu}(1-a u)^{v} d u$, abgesehen von einem constanten Factor, der hypergeometrischen Reihe gleich ist, welche nach Gaufs mit $\boldsymbol{F}^{\prime}(-\nu, \lambda+1, \lambda+\mu+2, a)$ bezeichnet wird. Ferner übersieht man leicht, dafs sich die Grenzen der sechs Integrale durch geeignete Substitutionen in 0 und 1 verwandeln lassen, ohne dafs die Function unter dem Integrale ihre Form $u^{p}(1-u)^{q}(1-b u)^{r} d u$ ver20 * 
liert. Ich stelle die sechs in hypergeometrische Reihen ausgedrückten Lösungen, auf die man so nach einander kommt, mit den Substitutionen zusammen, welche angewandt wurden:

1) $\boldsymbol{F}(\alpha, \beta, \gamma, x)$

2) $x^{-\alpha} \boldsymbol{F}\left(\alpha, \alpha+1-\gamma, \alpha+\beta+1-\gamma, \frac{x-1}{x}\right)$,

3) $x^{-\alpha} F\left(\alpha, \alpha+1-\gamma, \alpha+1-\beta, \frac{1}{x}\right)$,

4) $x^{-\beta} \boldsymbol{F}\left(\beta, \beta+1-\gamma, \beta+1-\alpha, \frac{1}{x}\right)$,

5) $\boldsymbol{x}^{1-\gamma} \boldsymbol{F}(\alpha+1-\gamma, \beta+1-\gamma, 2-\gamma, x)$,

6) $x^{\alpha-\gamma}(1-x)^{\gamma-\alpha-\beta} F\left(\gamma-\alpha, 1-\alpha, \gamma+1-\alpha-\beta, \frac{x-1}{x}\right)$,

Substitution $u=v$,

$$
\begin{aligned}
- & u & =\frac{v-1}{v}, \\
- & u & =\frac{1}{v} \\
- & u & =\frac{v}{x} \\
- & u & =\frac{1}{x v},
\end{aligned}
$$

Substitution $u=\frac{1}{x+(1-x) v}$.

$\mathrm{Zu}$ jeder dieser Lösungen findet man drei gleiche, nur der Form nach verschiedene, wenn man, nachdem die Grenzen der Integrale bereits durch obige Substitutionen auf 0 und 1 gebracht sind, noch drei neue Substitutionen anwendet, welche die Grenzen ungeändert lassen, nämlich die folgenden:

$$
u=1-v ; \quad u=\frac{v}{1-x+v x} ; \quad u=\frac{1-v}{1-v x} .
$$

Durch diese geht $V d u$ resp. in

$$
\begin{aligned}
& (1-x)^{-\alpha} v^{\gamma-\beta-1}(1-v)^{\beta-1}\left(1-\frac{x v}{x-1}\right)^{-\alpha} d v, \\
& (1-x)^{-\beta} v^{\beta-1}(1-v)^{\gamma-\beta-1}\left(1-\frac{x v}{x-1}\right)^{\alpha-\gamma} d v, \\
& (1-x)^{\gamma-\alpha-\beta} v^{\gamma-\beta-1}(1-v)^{\beta-1}(1-v x)^{\alpha-\gamma} d v
\end{aligned}
$$

über; sie führen also von $F(\alpha, \beta, \gamma, x)$ auf

$$
\begin{aligned}
& (1-x)^{-\alpha} F\left(\alpha, \gamma-\beta, \gamma, \frac{x}{x-1}\right) \\
& (1-x)^{-\beta} F\left(\gamma-\alpha, \beta, \gamma, \frac{x}{x-1}\right) \\
& (1-x)^{\gamma-\alpha-\beta} F(\gamma-\alpha, \gamma-\beta, \gamma, x) .
\end{aligned}
$$

Stellen wir nun die in hypergeometrische Reihen übertragenen Integrale zusammen, so haben wir sechs Klassen, von denen jede vier gleichbedeutende Lösungen enthălt: 
1) $\boldsymbol{F}(\alpha, \beta, \gamma, x)$,

\section{Classe I.}

2) $(1-x)^{\gamma-\alpha-\beta} \boldsymbol{F}(\gamma-\alpha, \gamma-\beta, \gamma, x)$,

3) $(1-x)^{-\alpha} \boldsymbol{F}\left(\alpha, \gamma-\beta, \gamma, \frac{x}{x-1}\right)$,

4) $(1-x)^{-\beta} F\left(\beta, \gamma-\alpha, \gamma, \frac{x}{x-1}\right)$.

\section{Classe II.}

1) $x^{-\alpha} \boldsymbol{F}\left(\alpha, \alpha+1-\gamma, \alpha+\beta+1-\gamma, \frac{x-1}{x}\right)$,

2) $x^{-\beta} \boldsymbol{F}\left(\beta, \beta+1-\gamma, \alpha+\beta+1-\gamma, \frac{x-1}{x}\right)$,

3) $\boldsymbol{H}(\alpha, \beta, \alpha+\beta+1-\gamma, 1-x)$,

4) $x^{1-\gamma} \boldsymbol{F}(\alpha+1-\gamma, \beta+1-\gamma, \alpha+\beta+1-\gamma, 1-x)$.

\section{Classe III.}

1) $x^{-\alpha} \boldsymbol{F}\left(\alpha, \alpha+1-\gamma, \alpha+1-\beta, \frac{1}{x}\right)$,

2) $x^{\beta-\gamma}(1-x)^{\gamma-\alpha-\beta} F\left(1-\beta, \gamma-\beta, \alpha+1-\beta, \frac{1}{x}\right)$,

3) $(1-x)^{-\alpha} H\left(\alpha, \gamma-\beta, \alpha+1-\beta, \frac{1}{1-x}\right)$,

4) $x^{1-\gamma}(1-x)^{\gamma-\alpha-1} F\left(\alpha+1-\gamma, 1-\beta, \alpha+1-\beta, \frac{1}{x}\right)$.

\section{Classe IV.}

1) $x^{-\beta} F\left(\beta, \beta+1-\gamma, \beta+1-\alpha, \frac{1}{x}\right)$,

2) $x^{\alpha-\gamma}(1-x)^{\gamma-\alpha-\beta} F\left(1-\alpha, \gamma-\alpha, \beta+1-\alpha, \frac{1}{x}\right)$,

3) $(1-x)^{-\beta} F\left(\beta, \gamma-\alpha, \beta+1-\alpha, \frac{1}{1-x}\right)$,

4) $x^{1-\gamma}(1-x)^{\gamma-\beta-1} F\left(\beta+1-\gamma, 1-\alpha, \beta+1-\alpha, \frac{1}{x}\right)$.

Classe V.

1) $x^{1-\gamma} \boldsymbol{F}(\alpha+1-\gamma, \beta+1-\gamma, 2-\gamma, x)$,

2) $x^{1-\gamma}(1-x)^{\gamma-\alpha-\beta} F(1-\alpha, 1-\beta, 2-\gamma, x)$,

3) $x^{1-\gamma}(1-x)^{\gamma-\alpha-1} F\left(\alpha+1-\gamma, 1-\beta, 2-\gamma, \frac{x}{x-1}\right)$,

4) $x^{1-\gamma}(1-x)^{\gamma-\beta-1} F\left(\beta+1-\gamma, 1-\alpha, 2-\gamma, \frac{x}{x-1}\right)$. 
Classe VI.
1) $x^{\alpha-\gamma}(1-x)^{\gamma-\alpha-\beta} F\left(\gamma-\alpha, 1-\alpha, \gamma+1-\alpha-\beta, \frac{x-1}{x}\right)$,
2) $x^{\beta-\gamma}(1-x)^{\gamma-\alpha-\beta} F\left(\gamma-\beta, 1-\beta, \gamma+1-\alpha-\beta, \frac{x-1}{x}\right)$,
3) $(1-x)^{\gamma-\alpha-\beta} F(\gamma-\alpha, \gamma-\beta, \gamma+1-\alpha-\beta, 1-x)$,
4) $x^{1-\gamma}(1-x)^{\gamma-\alpha-\beta} F(1-\alpha, 1-\beta, \gamma+1-\alpha-\beta, 1-x)$.

Diese 24 Reihen sind dieselben, welche Kummer im $\$$. 8 seiner Arbeit über die hypergeometrische Reihe im $15^{\text {ten }}$ Bande dieses Journals aufgeführt hat, über deren Bedeutung man an jener Stelle das Nähere findet. Die hier vorliegende Untersuchung giebt also das neue Resultat, dafs die bestimmten Integrale, die jenen Reihen gleich sind, sämmtlich durch Integration desselben Ausdrucks zwischen zweien der Grenzen $0,1, \pm \infty, \frac{1}{x}$ erhalten werden.

S. 2.

Eine ganz andere Art von Beziehungen zwischen Integralen der Gleichung (1.) erhält man durch Verallgemeinerung der Untersuchungen, welche Gau/s in seiner Arbeit über mechanische Quadraturen führt. Es tritt dort (art. 8) eine Function $\boldsymbol{T}$ vom $\boldsymbol{n}+1^{\text {ten }}$ Grade auf, deren Zusammenhang mit $\int_{0}^{1} \frac{T d t}{t-a}$ Anlafs zur Auffindung des folgenden Satzes gab:

Ist $y=f(x)$ ein Integral der Differentialgleichung (1.), so wird:

$$
\text { (2.) } \quad z=\int_{g}^{h} \frac{t \gamma-1(1-t)^{\alpha+\beta-\gamma}}{(t-x)^{\rho}} f(t) d t=\int_{g}^{h} W \cdot f t d t
$$

ein Integral der Differentialgleichung

(3.) $x(1-x) z^{\prime \prime}+(\varrho+1-\gamma-(2 \varrho+1-\alpha-\beta) x) z^{\prime}-(\rho-\alpha)(\varrho-\beta) z=0$, wenn sowohl $g$ als $h$ einen der Werthe $0,1, \pm \infty$ hat, und

$$
\left[\frac{t \gamma(1-i)^{\alpha+\beta+1-\gamma}}{(t-x)^{\rho}}\left(f^{\prime}(t)+\varrho \frac{f(t)}{t-x}\right)\right]_{g}^{h}=0
$$

ist. Man kann auch $h=x$ setzen; alsdann mufs aber der Ausdruck in der Parenthese für $\ell=g$ verschwinden, und $1-\varrho$ positiv sein.

Um diesen Satz zu beweisen, kann man davon ausgehen, dafs $f(t)$ der Differentialgleichung

$$
t(1-t) f^{\prime \prime}(t)+(\gamma-(\alpha+\beta+1) t) f^{\prime}(t)=\alpha \beta f(t)
$$


genügt, welche mit $t^{\gamma-1}(1-t)^{\alpha+\beta-\gamma}$ multiplicirt, die Form annimmt

$$
\alpha \beta t^{\gamma-1}(1-t)^{\alpha+\beta-\gamma} f(t)=\frac{d\left(t^{\gamma}(1-t)^{\alpha+\beta+1-\gamma} f^{\prime}(t)\right)}{d t} .
$$

Dies in das Integral auf der rechten Seite der Gleichung (2.) eingesetzt giebt nach einer theilweisen Integration:

$$
\alpha \beta z=\left[\frac{t \gamma(1-t)^{\alpha+\beta+1-\gamma} f^{\prime}(t)}{(t-x)^{\varrho}}\right]_{g}^{h}+\varphi \int_{g}^{h} \frac{t \gamma(1-t)^{\alpha+\beta+1-\gamma} f^{\prime}(t)}{(t-x)^{\rho+1}} d t
$$

und nach einer zweiten theilweisen Integration:

$$
\alpha \beta z=\left[t(1-t) W\left(f^{\prime}(t)+\frac{\rho f(t)}{t-x}\right)\right]_{g}^{h}-\varphi_{g}^{\rho} \frac{d}{d t}\left(\frac{t(1-t)}{t-x} \cdot W\right) f(t) d t .
$$

Wendet man nun die in der Anmerkung des $\$$. 1 gegebene Transformation an, nach welcher, wenn $u=\frac{1}{t}, u^{2} V=W$ gesetzt wird,

$$
\begin{gathered}
-\varrho \frac{d}{d t}\left(\frac{t(1-t)}{t-x} W\right) \\
=x(1-x) \frac{d^{2} W}{d x^{2}}+(\varrho+1-\gamma-(2 \varrho+1-\alpha-\beta) x) \frac{d W}{d x}-\rho(\rho-\alpha-\beta) W
\end{gathered}
$$

ist, so findet man den oben aufgestellten Satz.

Das in demselben enthaltene Ergebnifs läfst sich in eine andere Form bringen, und zwar durch Vergleichung der beiden Lösungen $F(\alpha, \beta, \gamma, x)$ und $x^{1-\gamma}(1-x)^{\gamma-\alpha-\beta} F(1-\alpha, 1-\beta, 2-\gamma, x)$ der Gleichung (1.), welche in den Ausdrücken 1) der $\mathrm{I}^{\text {ten }}$ Classe und 2) der $\mathrm{V}^{\text {ten }}$ Classe gegeben sind. Da nämlich $\boldsymbol{F}(1-\alpha, 1-\beta, 2-\gamma, x)$ eine Lösung $\zeta$ von

$$
\text { (1. a) } x(1-x) \zeta^{\prime \prime}+(2-\gamma \bar{F}(3-\alpha-\beta) x) \zeta^{\prime}-(1-\alpha)(1-\beta) \zeta=0
$$

ist, so läfst sich das Resultat jener Vergleichung in der Art aussprechen, dafs eine Lösung $\zeta$ von $(1 . a)$ multiplicirt mit $x^{1-\gamma}(1-x)^{\gamma-\alpha-\beta}$ eine Lōsung von (1.) giebt. Läfst man an die Stelle von (1.a) die Gleichung (3.) treten, indem man $\alpha, \beta, \gamma$ um 1 - $\varrho$ vermehrt, so folgt mit Hülfe des obigen Satzes unmittelbar, dafs

$$
\text { (4.) } \quad \mathbf{Z}=x^{\rho-\gamma}(1-x)^{\rho+\gamma-\alpha-\beta-1} \int_{g}^{h} \frac{t \gamma-1(1-t)^{\alpha+\beta-\gamma}}{(t-x)^{\rho}} f(t) d t
$$

eine Lösung derjenigen Gleichung wird, in welche gleichzeitig (1.) übergeht, d. h. von

(5.) $x(1-x) Z^{\prime \prime}+(\gamma+1-\rho-(\alpha+\beta+3-2 \varrho) x) Z^{\prime}-(\alpha+1-\rho)(\beta+1-\varrho) Z=0$. 
Setzt man hier ins Besondere $\varrho=1$, so wird (5.) mit (1.) identisch, und man erhält aus einem Integrale $f(x)$ der Gleichung (1.) ein zweites:

(6.) $x^{1-\gamma}(1-x)^{\gamma-\alpha-\beta} \int_{g}^{h} \frac{t^{\gamma-1}(1-t)^{\alpha+\beta-\gamma}}{t-x} f(t) d t$.

\$. 3.

Die letzte Formel giebt ein besonders interessantes Resultat, wenn $f(t)$ eine endliche Reihe, also eine solche hypergeometrische Reihe ist, deren erstes oder zweites Element eine negative ganze Zahl $-n$ wird. Ueber die Eigenschaften solcher endlichen Reihen soll in diesem und den folgenden Paragraphen bis $\$ .6$ einschliefslich gehandelt werden.

Differentiirt man die Gleichung (1.)

$$
x(1-x) y^{\prime \prime}+(\gamma-(\alpha+\beta+1) x) y^{\prime}-\alpha \beta y=0
$$

mehrere Male hintereinander nach $x$, so erhält man

$$
\begin{gathered}
x(1-x) y^{\prime \prime \prime}+(\gamma+1-(\alpha+\beta+3) x) y^{\prime \prime}-(\alpha+1)(\beta+1) y^{\prime}=0 \\
x(1-x) y^{\prime \prime}+(\gamma+2-(\alpha+\beta+5) x) y^{\prime \prime \prime}-(\alpha+2)(\beta+2) y^{\prime \prime}=0 \\
\text { etc. } \quad \text { etc. }
\end{gathered}
$$

Das durch (n-1)malige Differentiation gewonnene Resultat wird durch Multiplication mit

$$
x^{\gamma+n-2}(1-x)^{\alpha+\beta-\gamma+n-1}
$$

auf die Form gebracht

$$
\frac{d\left(x^{n}(1-x)^{n} M y^{(n)}\right)}{d x}=(\alpha+n-1)(\beta+n-1) x^{n-1}(1-x)^{n-1} M y^{(n-1)},
$$

wo

$$
\boldsymbol{M}=x^{\gamma-1}(1-x)^{\alpha+\beta-\gamma} .
$$

Indem man diese Gleichung noch ferner $(n-1)$ mal differentiirt, erhält man

$$
\frac{d^{n}\left\{x^{n}(1-x)^{n} M y^{(n)}\right\}}{d x^{n}}=(\alpha+n-1)(\beta+n-1) \frac{d^{n-1}\left\{x^{n-1}(1-x)^{n-1} M\right.}{d x^{n-1}} \underline{\left.y^{(n-1)}\right\}},
$$

und durch wiederholte Anwendung ergiebt sich hieraus für jedes ganze positive $n$ die Gleichung:

$$
\frac{d^{n}\left(x^{n}(1-x)^{n} M y^{(n)}\right)}{d x^{n}}=\alpha(\alpha+1) \ldots(\alpha+n-1) \cdot \beta \cdot(\beta+1) \ldots(\beta+n-1) M y,
$$

in welcher $M$ denselben Werth wie oben bezeichnet.

Ist nun $y$ eine bei der nten Potenz von $x$ abbrechende hypergeometrische 
Reihe, setzt man also $\beta=-n$, während $\alpha$ und $\gamma$ beliebig bleiben, so wird

$$
\boldsymbol{y}=\boldsymbol{F}(-n, \alpha, \gamma, \boldsymbol{x})
$$

und nach obiger Gleichung:

$$
\boldsymbol{F}(-n, \alpha, \gamma, x)=\frac{x^{1-\gamma}(1-x)^{\gamma+n-\alpha}}{\gamma(\gamma+1) \ldots(\gamma+n-1)} \frac{d^{n}\left(x^{\gamma+n-1}(1-x)^{\alpha-\gamma}\right)}{d x^{n}}
$$

oder, wenn man $\alpha+n$ für $\alpha$ setzt,

$$
\boldsymbol{F}^{\prime}(-n, \alpha+n, \gamma, x)=\frac{x^{1-\gamma}(1-x)^{\gamma-\alpha}}{\gamma(\gamma+1) \ldots(\gamma+n-1)} \frac{d^{n}\left(x^{\gamma+n-1}(1-x)^{\alpha+n-\gamma}\right)}{d x^{n}} .
$$

Dieser Ausdruck zeigt einerseits, dafs sich jede endliche hypergeometrische Reibe in die elegante Form der rechten Seite von (7.) bringen läfst, und giebt andrerseits dem häufig vorkommenden Differentialausdrucke der rechten Seite die entwickelte Form eines Productes von Potenzen in eine einfache hypergeometrische Reihe. Für $\alpha=\gamma=1$ erhält man

$$
\frac{1}{1.2 \ldots n} \frac{d^{n}\left(x^{n}(1-x)^{n}\right)}{d x^{n}}=\boldsymbol{F}(-n, n+1,1, x)
$$

und setzt man $x=\frac{1-\xi}{2}$

$$
\frac{1}{2^{n} .1 .2 \ldots n} \frac{d^{n}\left(\xi^{2}-1\right)^{n}}{d \xi^{n}}=F\left(-n, n+1,1, \frac{1-\xi}{2}\right),
$$

also links die bekannte Function, welche durch Entwicklung von $\frac{1}{\sqrt{1-2 h \xi+h^{2}}}$ nach Potenzen von $h$ entsteht. Den Ausdruck derselben durch die Reihe auf der rechten Seite findet man bei Dirichlet (Bd. 17, S. 39 dieses Journals). Auf ähnliche Art erbält man eine Entwicklung des nten Differentialquotienten $\frac{d^{n}\left(1-\xi^{2}\right)^{n-\frac{\pi}{2}}}{d \xi^{n}}$, welcher bekanntlich für $\xi=\cos \varphi$ mit $\cos n \varphi$ zusammenhängt.

S. 4.

Es macht keine Schwierigkeit, die erzeugende Function der durch (7.) gegebenen Ausdrücke in derselben Art aufzufinden, wie es im zweiten Bande dieses Journals S. 224 in dem schon $\$$. 3 erwähnten besonderen Falle $\alpha=\gamma=1$ geschehen ist. Man kann sich dazu der Lagrangeschen Formel bedienen, nach der

$$
\chi(y) \frac{d y}{d x}=\chi(x)+\frac{h}{1} \frac{d(f(x) \chi(x))}{d x}+\frac{h^{2}}{1.2} \frac{d^{2}\left(f(x)^{2} \chi(x)\right)}{d x^{2}}+\text { elc. }
$$

ist, wenn zwischen $x$ und $\boldsymbol{y}$ die Gleichung:

$$
y-x=h f(y)
$$


besteht, und hat nur

$$
f(x)=x(1-x) ; \quad \chi(x)=x^{\gamma-1}(1-x)^{\alpha-\gamma}
$$

zu setzen. Macht man

$$
\boldsymbol{F}(-\boldsymbol{n}, \alpha+\boldsymbol{n}, \gamma, \boldsymbol{x})=\boldsymbol{X}_{n}
$$

und wie oben $2 x=1-\xi$, so erhält man

$$
\begin{gathered}
\frac{x^{1-\gamma}(1-x)^{\gamma-\alpha}\left\{h-1+\sqrt{1-2 h \xi+h^{2}}\right\}^{\gamma-1}\left\{h+1-\sqrt{1-2 h \xi+h^{2}}\right\}^{\alpha-\gamma}}{(2 h)^{\alpha-1} \sqrt{1-2 h \xi+h^{2}}} \\
=\sum_{n=0}^{n=\infty} \frac{\gamma(\gamma+1) \ldots(\gamma+n-1)}{1.2 \ldots n} h^{n} \boldsymbol{X}_{n} .
\end{gathered}
$$

Diese Formel, welche sich nicht durch Einfachheit zu empfehlen schien, ist nicht weiter verfolgt worden, sondern zunächst nur ein besonderer Fall derselben.

S. 5 .

Man entwickle nämlich mit Hülfe des binomischen Lehrsatzes

$$
\left(1-2 h \xi+h^{2}\right)^{-c}
$$

nach aufsteigenden Potenzen von h. Setzt man die so entstehende Reihe

so wird

$$
=\sum_{n=0}^{n=\infty} h^{n} \boldsymbol{Y}_{n}
$$

$$
\boldsymbol{Y}_{n}=\frac{2 c(2 c+1) \ldots(2 c+n-1)}{1.2 \ldots n} \boldsymbol{H}\left(-n, 2 c+n, \frac{2 c+1}{2}, x\right),
$$

wenn $\boldsymbol{x}$ und $\boldsymbol{\xi}$ wie im vorigen Paragraphen zusammenhangen, also auch

$$
Y_{n}=4^{n} \frac{c(c+1) \ldots(c+n-1)(x(1-x))^{\frac{1}{(1-2 c)}}}{(2 c+n)(2 c+n+1) \ldots(2 c+2 n-1) I n} \frac{d^{n}(x(1-x))^{\frac{1}{2}(2 c+2 n-1)}}{d x^{n}} .
$$

\$. 6 .

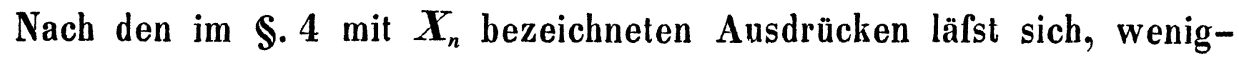
stens so lange $\gamma$ und $\alpha+1-\gamma$ positiv sind, eine Function $\varphi(x)$ nur auf eine Art entwickeln, so dafs also, wenn man $\varphi(x)=\sum_{n=0}^{n=\infty} a_{n} X_{n}$ setzt, die Constanten a vollständig bestimmt sind. Man hat zum Beweise dieses Satzes nur zu zeigen, dafs

$$
J_{m, n}=\int_{0}^{1} \boldsymbol{X}_{m} \boldsymbol{X}_{n} x^{\gamma-1}(1-x)^{\alpha-\gamma} d x
$$

verschwindet, sobald die ganzen Zahlen $m$ und $n$ von einander verschieden 
sind. Es genügt aber $\boldsymbol{X}_{n}$ der Differentialgleichung

so dafs

$$
x(1-x) X_{n}^{\prime \prime}+(\gamma-(\alpha+1) x) \boldsymbol{X}_{n}^{\prime}=-n(n+\alpha) \boldsymbol{X}_{n},
$$

$$
\begin{aligned}
-\boldsymbol{n}(\boldsymbol{n}+\alpha) \boldsymbol{J}_{m, n} & =\int_{0}^{1} \boldsymbol{X}_{m} \frac{d\left(x^{\gamma}(1-x)^{\alpha+1-\gamma} X_{n}^{\prime}\right)}{d x} d x \\
& =\int_{0}^{1} \boldsymbol{X}_{n} \frac{d\left(x^{\gamma}(1-x)^{\alpha+1-\gamma} X_{m}^{\prime}\right)}{d x} d x
\end{aligned}
$$

also gleich $-\boldsymbol{m}(\boldsymbol{m}+\alpha) \boldsymbol{J}_{m, n}$ wird, woraus man schliefst, dafs $\boldsymbol{J}_{m, n}$ verschwindet. Ist $m=n$, so läfst sich der Werth dieser Constanten leicht angeben, da offenbar

$$
\boldsymbol{n}(\boldsymbol{n}+\alpha) \boldsymbol{J}_{n, n}=\int_{U}^{1} \boldsymbol{X}_{n}^{\prime} \boldsymbol{X}_{n}^{\prime} \boldsymbol{x}^{\gamma}(1-\boldsymbol{x})^{a+1-\gamma} d \boldsymbol{x}
$$

ist, ferner

$$
\begin{aligned}
& (n-1)(n+\alpha+1) \int_{0}^{1} X_{n}^{\prime} X_{n}^{\prime} x^{\gamma}(1-x)^{\alpha+1-\gamma} d x=\int_{0}^{1} X_{n}^{\prime \prime} X_{n}^{\prime \prime} x^{\gamma+1}(1-x)^{\alpha+2-\gamma} d x \\
& \text { etc., }
\end{aligned}
$$

so dafs man für $\boldsymbol{J}_{n, n}$ den Werth

erbält.

$$
\frac{1}{\alpha+2 n} \frac{\Pi n(\Pi(\gamma-1))^{2} \Pi(\alpha+n-\gamma)}{\Pi(\alpha+n-1) \Pi(\gamma+n-1)}
$$

\section{\$. 7.}

Für ein zweites Integral der Differentialgleichung, deren erstes $\boldsymbol{X}_{n}$ ist, erhält man durch die Formel (6.) des $\$$. 2 den Werth

$$
x^{1-\gamma}(1-x)^{\gamma-\alpha} \int_{g}^{h} \frac{t^{\gamma-1}(1-t)^{\alpha-\gamma}}{(t-x)} F(-n, \alpha+n, \gamma, t) d t,
$$

welcher für $\alpha=\gamma=1$ in den am Anfange des $\$ .2$ erwähnten übergeht, wenn man $n+1$ für $n$, und $g=0, h=1$ setzt.

Nach $\$ .3$ kann der obige Werth auch durch

$$
x^{1-\gamma}(1-x)^{\gamma-\alpha} \int_{g}^{h} \frac{d^{n}\left(t^{\gamma+n-1}(1-t)^{\alpha+n-\gamma}\right)}{d t^{n}} \frac{d t}{t-x}
$$

ersetzt werden, also auch, wenn die Werthe $\gamma, \alpha$ eine Integration durch Theile gestatten, durch

(8.) $\quad Z_{n}=x^{1-\gamma}(1-x)^{\gamma-\alpha} \int_{g}^{h} \frac{t^{\gamma+n-1}(1-t)^{\alpha+n-\gamma}}{(t-x)^{n+1}} d t$. 
Die Differentialgleichung ist dann durch

$$
\boldsymbol{a} \boldsymbol{X}_{n}+\mathbf{b} \mathbf{Z}_{n}
$$

vollständig integrirt, wenn $a$ und $b$ willkürliche Constanten bezeichnen.

\$. 8.

Die Resultate, welche Gaurs durch Vergleichung von $T$ und $\int_{0}^{1} \frac{T d t}{t-a}$ für die Kettenbruchentwicklung der logarithmischen Reihe gefunden hat, lassen sich durch Vergleichung von $\boldsymbol{X}_{n}$ und $\boldsymbol{Z}_{n}$ auf die besondere hypergeometrische Reihe $\boldsymbol{F}(\alpha, 1, \gamma, x)$ übertragen. Man erhält auf diesem Wege fast ohne Rechnung die Resultate über die Werthe der Näherungsbrüche von Kettenbrüchen, die zuerst durch Auflösung linearer Gleichungen gefunden worden sind (Bd. 32, S. 208, so wie Bd. 34, S. 297 dieses Journals).

Es seien $\alpha+1-\gamma$ und $\gamma$ positiv, ferner $x>1$; bezeichnet man den Werth von $\boldsymbol{X}_{n}$ für $x=t$ mit $\boldsymbol{T}_{n}$ und setzt

$$
-W_{n}=\int_{0}^{1} t^{\gamma-1}(1-t)^{\alpha-\gamma} \frac{T_{n}-X_{n}}{t-x} d t,
$$

so dafs $\boldsymbol{W}_{n}$ eine ganze Function $(n-1)^{\text {ten }}$ Grades von $x$ ist, so hat man offenbar die Gleichung

$$
X_{n} \int_{0}^{1} \frac{t \gamma-1(1-t)^{\alpha-\gamma}}{(t-x)} d t=W_{n}+\int_{0}^{1} \frac{t^{\gamma-1}(1-t)^{\alpha-\gamma}}{t-x} T d t
$$

und hieraus, wenn man mit $a$ und $b$ leicht zu berechnende Constanten bezeichnet,

$$
\frac{a}{x} X_{n} F\left(\gamma, 1, \alpha+1, \frac{1}{x}\right)=W_{n}+b \int_{0}^{1} \frac{t \gamma+n-1(1-t)^{\alpha+n-\gamma}}{(t-x)^{n+1}} d t .
$$

Das mit $b$ multiplicirte Integral, nach absteigenden Potenzen von $x$ entwickelt, fängt mit $x^{-n-1}$ an (der Grad ist $-(n+1)$ ): wir haben also eine Function $\boldsymbol{n}^{\text {ten }}$ Grades $\boldsymbol{X}_{n}$, die, mit $\boldsymbol{F}\left(\gamma, 1, \alpha+1, \frac{1}{x}\right)$ multiplicirt, eine ganze Function $\boldsymbol{x} \boldsymbol{W}_{n}$ und einen Rest vom $-\boldsymbol{n}^{\text {ten }}$ Grade giebt. Seit der Arbeit von Gau/s über mechanische Quadraturen ist es bekannt, wie diese Eigenschaft $\operatorname{der} \boldsymbol{X}_{n}$ es möglich macht, sofort die Nenner der Näherungsbrüche des Kettenbruches für $\boldsymbol{F}\left(\alpha, 1, \gamma, \frac{1}{x}\right)$, wie er sich aus der Abhandlung von Gau/s über die hyper- 
geometrische Reihe (art.13) ergiebt, nämlich nach der dortigen Bezeichnung von

$$
\frac{x}{x-\frac{a}{1-\frac{b}{x-c}}}
$$

anzugeben. Der Nenner des $2 n^{\text {ten }}$ Näherungswerthes $\boldsymbol{Q}_{2 n}$ ist nämlich von der Form

$\operatorname{der} \operatorname{des}(2 n+1)^{\text {ten }}$

$$
\boldsymbol{Q}_{2 n}=x^{n}+b_{1} x^{n-1}+b_{2} x^{n-2}+\cdots+b_{n},
$$

$$
\boldsymbol{Q}_{2 n+1}=x\left(x^{n}+c_{1} x^{n-1}+c_{2} x^{n-2}+\cdots+c_{n}\right),
$$

wenn wir $x$ als den ersten, $x-a$ als den zweiten zählen. Ferner mufs $\boldsymbol{Q}_{2 n}$ oder $\boldsymbol{Q}_{2 n+1}$, mit $\boldsymbol{H}\left(\alpha, 1, \gamma, \frac{1}{x}\right)$ multiplicirt, gleich einer ganzen Function von $x$ vermehrt um einen Rest vom Grade $-n$ sein. Es können sich daher $\boldsymbol{Q}_{2 n}$ und $\boldsymbol{Q}_{2 n+1}$ nur durch constante Factoren von $\boldsymbol{F}(-n, \gamma+n-1, \alpha, x)$ und $x \boldsymbol{F}(-n, \gamma+n, \alpha+1, x)$ unterscheiden; bestimmt man diese gehörig, nämlich so, dafs die höchste Potenz von $x$ die Einheit zum Factor erhält, so entsteht

$$
\begin{aligned}
\boldsymbol{Q}^{2 n} & =x^{n} \boldsymbol{F}\left(-n, 1-\alpha-n, 2-\gamma-2 n, \frac{1}{x}\right), \\
\boldsymbol{Q}_{2 n+1} & =x^{n+1} \boldsymbol{F}\left(-n,-\alpha-n, 1-\gamma-2 n, \frac{1}{x}\right) .
\end{aligned}
$$

Haben $\gamma$ und $\alpha+1-\gamma$ andere Zeichen, so kann sich an den Resultaten offenbar nichts ändern.

\section{\$. 9.}

Wir gehen nun zu der letzten Untersuchung über, nämlich zur Beantwortung der Frage, ob es für jeden endlichen Werth der Elemente möglich ist, die Differentialgleichung (1.) durch einfache bestimmte Integrale vollständig zu integriren. Dafs die sechs bestimmten Integrale des $\$$. 1 nicht zugleich gelten, sieht man ohne weiteres ein; indem ich nun nicht nur, wie früher,

sondern auch

$$
\boldsymbol{V}=u^{\beta-1}(1-u)^{\gamma-\beta-1}(1-x u)^{-\alpha},
$$

$$
W=u^{\alpha-1}(1-u)^{\gamma-\alpha-1}(1-x u)^{-\beta}
$$

setze, sollen in der folgenden Tabelle die Fälle angegeben werden, in denen $\int V d u$ oder $\int W d u$ eine Lösung von (1.) verschafft. Es ist dabei nach den Vorzeichen von $\alpha, \beta, \gamma-\alpha, \gamma-\beta$ eingetheilt und, um die Anzahl der Fälle zu beschränken, angenommen worden, dafs $\beta-\alpha$ nicht negativ ist. 


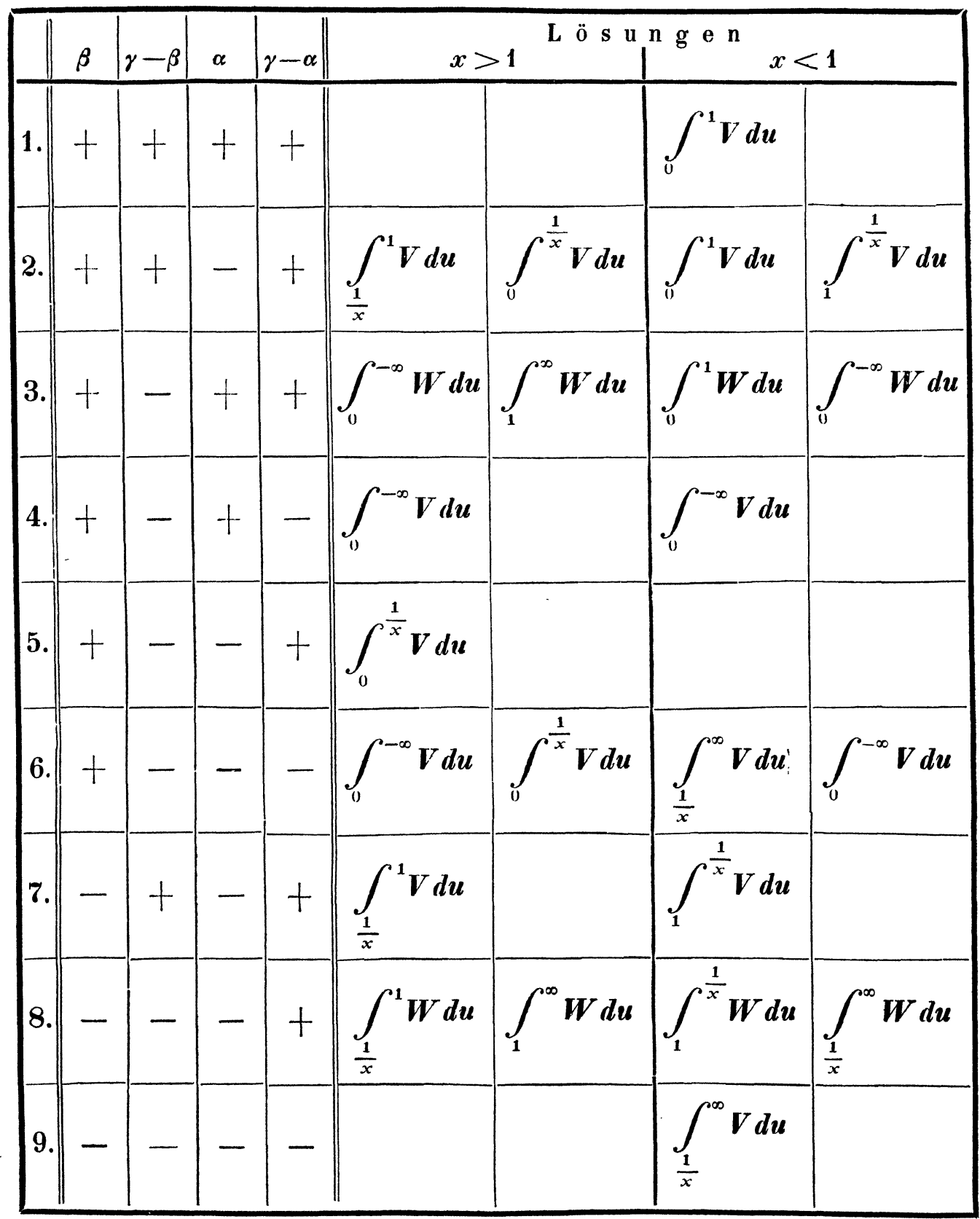

Da sowohl für den Fall, dafs $x>1$ als auch für den, dafs $x<1$, zwei verschiedene Lösungen angegeben werden müssen, so erkennt man in der Tabelle leicht, wann noch Lösungen zu suchen sind. 


\section{\$. 10.}

Um solche aufzufinden, kann man sich des in $\$$. 2 aufgestellten Satzes bedienen. Dieser giebt nämlich die Beziehungen zwischen den Differentialgleichungen zweier hypergeometrischen Reihen, deren Elemente $\alpha, \beta, \gamma$ und $\varrho-\alpha, \varrho-\beta, \varrho+1-\gamma$ sind. Man wende ihn an, indem man an die Stelle von $\alpha, \beta, \gamma$ respective $\rho-\alpha, \varrho-\beta, \rho+1-\gamma$ setzt, so dafs zugleich $\varrho-\alpha$, $\rho-\beta, \rho+1-\gamma$ respeclive in

$$
\varphi-(\varphi-\alpha)=\alpha, \quad \varrho-(\varphi-\beta)=\beta, \quad \varphi+1-(\varphi+1-\gamma)=\gamma
$$

übergehen. Nimmt man nun $\varrho$ so, dafs $\rho-\alpha$ gleich einer negativen ganzen Zahl $-n$ wird, so ist ein Integral der ersten Differentialgleichung eine endliche Reihe

$$
\boldsymbol{F}(-\boldsymbol{n}, \alpha-\beta-n, \alpha+1-\gamma-n, x)=f(x) ;
$$

die zweite Differentialgleichung ist jetzt die Differentialgleichung (1.) selbst, nach (2.) findet man also ein Integral von (1.) durch die Formel:

$$
\Xi=\int_{g}^{h} \frac{t^{\alpha-\gamma-n}(1-t)^{\gamma-\beta-n-1}}{(t-x)^{\alpha-n}} f(t) d t
$$

oder mit Benutzung der in $\$ .3$ gegebenen Umformung durch die Formel:

$$
\text { (9.) } \quad \mathbf{Z}=\int_{g}^{h} \frac{d^{n}\left\{t^{\alpha-\gamma}(1-t)^{\gamma-\beta-1}\right\}}{d t^{n}}(t-x)^{n-\alpha} d t \text {, }
$$

vorausgesetzt, dafs bei constantem $g$ und $h$

$$
\left[\frac{t^{\alpha+1-\gamma-n}(1-t)^{\gamma-\beta-n}}{(t-x)^{\alpha-n}}\left(f^{\prime}(t)+\frac{\alpha-n}{t-x} f(t)\right)\right]_{g}^{h}=0
$$

ist, und dafs, wenn $h=x$, der Ausdruck in der Parenthese für $t==g$ verschwindet und $n+1-\alpha$ positiv ist.

Dieses Resultat läfst sich übrigens leicht verificiren, und ähnliche lassen sich eben so leicht auffinden, wenn man erwägt, dafs nach Integration durch Theile auf der rechten Seite unter dem Integrale

$$
t^{\alpha-\gamma}(1-t)^{\gamma-\beta-1}(t-x)^{-\alpha} d t
$$

übrig bleibt, wenn diese Operation erlaubt ist. Dieser Ausdruck, zwischen $g$ und $h$ integrirt, ist aber eine Lösung von (1.); sind z. B. $g$ und $h$ gleich 0 und 1, so giebt die Integration eine Lösung der dritten Klasse. Man schliefst hieraus unmittelbar, dafs auch, wenn die Integration durch Theile nicht gestattet ist, $\mathbb{Z}$ eine Lösung von (1.) ist, wenn nur das Integral einen Werth hat. 


\section{\$. 11.}

Wir können nun die Tafel des $\$ 9.9$ vervollständigen.

1) Im ersten Falle fehlen zwei Integrale, wenn $x>1$; man kann offenbar foly ende hinzufügen:

$$
\begin{aligned}
& \int_{x}^{\infty} \frac{d^{n}\left\{t^{\beta-\gamma}(1-t)^{\gamma-\alpha-1}\right\}}{d t^{n}}(t-x)^{n-\beta} d t, \\
& \int_{x}^{\infty} \frac{t^{n}\left\{t^{\alpha-\gamma}(1-t)^{\gamma-\beta-1}\right\}}{d t^{n}}(t-x)^{n-\alpha} d t,
\end{aligned}
$$

wenn $n$ so grofs genommen wird, da's respective $n+1-\beta$ oder $n+1-\alpha$ positiv ist. Darf $n=0$ genommen werden, so sind diese Integrale respective von der III $^{\text {ten }}$ und IV $^{\text {ten }}$ Klasse.

Ist $x<1$, so fehlt ein Integral, welches man jedenfalls $=(1-x)^{-\beta} \zeta$ setzen kann, wo $\zeta$ der Differentialgleichung (1.) genügt, wenn man in ihr $\alpha, \beta, \gamma, x$ mit $\beta, \gamma-\alpha, \beta+1-\alpha, \frac{1}{1-x}$ vertauscht. Man vergl. Form 3) der IV $^{\text {ten }}$ Classe. Hieraus folgt, dafs als fehlendes Integral

$$
(1-x)_{\frac{1}{1-x}}^{-\beta} \int^{\infty} \frac{d^{n}\left\{t \gamma-\beta-1(1-t)^{-\alpha}\right\}}{d t^{n}}\left(t-\frac{1}{1-x}\right)^{\alpha+n-\gamma} d t
$$

beirachtel werden kann, wenn $\alpha+n+1-\gamma$ positiv ist. Für $n=0$ erhält a. $n$ ein Integral $\mathbf{I I}^{\text {ter }}$ Classe.

2) Im $4^{\text {ten }}$ Falle, wenn $x>1$, kann offenbar

$$
\int_{x}^{\infty} \frac{d^{n}\left\{t^{\beta-\gamma}(1-t)^{\gamma-\alpha-1}\right\}}{d t^{n}}(t-x)^{n-\beta} d t
$$

mit der Bedingung, dafs $n+1-\beta$ positiv ist, als Lösung genommen werden. Ist $x<1$, so mache man mit Beachtung der Form 1) der II $^{\text {ten }}$ Classe das fehlende Integral $=x^{-\alpha} \zeta$, wo $\zeta$ der Differentialgleichung genügt, in welche (1.) übergeht, wenn für $\alpha, \beta, \gamma, x$ resp. $\alpha, \alpha+1-\gamma, \alpha+\beta+1-\gamma, \frac{x-1}{x}$ gesetzt wird. Dadurch erhält man als Lösung

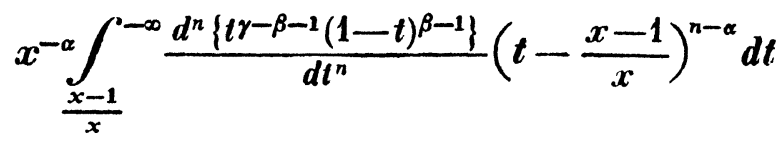

mit der Bedingung, dafs $n+1-\alpha$ positiv sein mufs. Für $n=0$ erhält man Integrale der III $^{\text {ten }}$ und $\mathrm{V}^{\text {ten }}$ Classe. 
3) Im fünften Falle mufs man ein Integral suchen, wenn $x>1$. Mit Berücksichtigung des $4^{\text {ten }}$ Integrals $I^{\text {ter }}$ Classe setze man $z=(1-x)^{-\beta} \zeta$, und vertausche ähnlich wie oben $\alpha, \beta, \gamma, x$ mit $\gamma-\alpha, \beta, \gamma, \frac{x}{x-1}$, so findet man

$$
(1-x)_{\frac{x}{x-1}}^{-\beta} \int^{\infty} \frac{d^{n}\left\{t \beta-\gamma(1-t)^{\alpha-1}\right\}}{d t^{n}}\left(t-\frac{x}{x-1}\right)^{n-\beta} d t
$$

mit der Bedingung, dafs $n+1-\beta$ positiv sei. Ist $x<1$, so erhält man zwei Integrale

$$
\begin{gathered}
(1-x)^{-\beta} \int_{\frac{x}{x-\infty}}^{-\infty} \frac{d^{n}\left\{t \beta-\gamma(1-t)^{\alpha-1}\right\}}{d t^{n}}\left(t-\frac{x}{x-1}\right)^{n-\beta} d t, \\
x^{-\beta} \int_{\frac{1}{x}}^{\infty} \frac{d^{n}\left\{t^{\alpha-\gamma}(1-t)^{-\alpha}\right\}}{d l^{n}}\left(t-\frac{1}{x}\right)^{\gamma+n-\beta-1} d t
\end{gathered}
$$

resp. mit den Bedingungen, dafs $n+1-\beta$ und $\gamma+n-\beta$ positiv sein müssen. Für $n=0$ verwandeln sich die Integrale in solche der $\mathrm{VI}^{\text {ten }} ; \mathrm{VI}^{\text {ten }}$ und $I^{\text {ten }}$ Classe.

4) Im $7^{\text {ten }}$ Falle findet man für $x>1$

$$
x^{1-\gamma}(1-x)^{\gamma-\alpha-\beta} \int_{x}^{\infty} \frac{d^{n}\left\{t \gamma-\beta-1(1-t)^{\alpha-\gamma\}}\right.}{d t^{n}}(t-x)^{n+\beta-1} d t
$$

und für $x<1$

$$
x^{\alpha-\gamma}(1-x)^{\gamma-\alpha-\beta} \int_{\frac{x-1}{x}}^{-\infty} \frac{d^{n}\left\{t \beta-\gamma(1-t)^{-\beta}\right\}}{d t^{n}}\left(t-\frac{x-1}{x}\right)^{n+\alpha-1} d t
$$

resp. mit den Bedingungen, dafs $n+\beta$ und $n+\alpha$ positiv sein müssen. Für $n=0$ entstehen Integrale der $I^{\text {ten }}$ und $I^{\text {ten }}$ Classe.

5) Im $9^{\text {ten }}$ Falle erhält man für $x>1$

und

$$
x^{1-\gamma} \int_{x}^{\infty} \frac{d^{n}\left\{t^{\beta-1}(1-t)^{-\alpha}\right\}}{d t^{n}}(t-x)^{\gamma+n-\beta-1} d t
$$

endlich für $x<1$

$$
x^{1-\gamma} \int_{x}^{\infty} \frac{d^{n}\left\{t^{\alpha-1}(1-t)^{-\beta}\right\}}{d t^{n}}(t-x)^{\gamma+n-\alpha-1} d t
$$

$$
x^{1-\gamma}(1-x)^{\gamma-\beta-1} \int_{\frac{1}{1-x}}^{\infty} \frac{d^{n}\left\{t-\beta(1-t)^{\gamma-\alpha-1}\right\}}{d t^{n}}\left(t-\frac{1}{1-x}\right)^{n+\alpha-1} d t .
$$

Die Bedingungen sind resp., dafs $\gamma+n-\beta, \gamma+n-\alpha$ und $n+\alpha$ positiv sein müssen; für $n=0$ entstehen Integrale der III $^{\text {ten }}$, IV $^{\text {ten }}$ und. II ${ }^{\text {ten }}$ Classe. 